\title{
Indole-3-Carbinol Induces Apoptosis of Hepatic Stellate Cells through K63 De- Ubiquitination of RIP1 in Rats
}

\author{
Bin Lia,b Meng Cong ${ }^{a}$ Yanan Zhu ${ }^{a} \quad$ Ying Xiong ${ }^{a}$ Wenyi Jin ${ }^{a}$ Yang Wan ${ }^{a}$ \\ Yunjiao Zhou ${ }^{\mathrm{a}}$ Ying Ao $\mathrm{A}^{\mathrm{a}, \mathrm{c}}$ Hui Wanga,c \\ aDepartment of Pharmacology, School of Basic Medical Science of Wuhan University, Wuhan, \\ ${ }^{b}$ Department of Orthopedic Surgery, Zhongnan Hospital of Wuhan University, Wuhan, \\ cHubei Provincial Key Laboratory of Developmentally Originated Disorder, Wuhan, China
}

\section{Key Words}

Indole-3-carbinol $\bullet$ Hepatic stellate cells $\bullet$ Receptor-interacting protein $1 \bullet \mathrm{K} 63$ de-ubiquitination - Cylindromatosis

\begin{abstract}
Background/Aims: The apoptosis of activated hepatic stellate cells (HSCs) is the central event in the reversal of liver fibrosis. K63 de-ubiquitinated receptor-interacting protein (RIP)1 promotes apoptosis in tumor necrosis factor (TNF)- $\alpha$ signaling pathway. In the previous study, we have proved that indole-3-carbinol (I3C) could reverse different models of liver fibrosis in rats, but the mechanism is still unclear. Thus, the present research aimed to demonstrate the induction of I3C on apoptosis of HSCs and the underlying molecular mechanism. Methods: HSC-T6, an immortalized rat liver stellate cell line, was treated for 24 hours with 25, 50 and $100 \mu \mathrm{M}$ of I3C. The apoptosis of HSC-T6 was analyzed by flow cytometric analysis, acridine orange staining and RT-PCR, respectively. K63 de-ubiquitination of RIP1 and the expression of ubiquitin ligases and deubiquitinases were analyzed by Co-IP assay and western blot. Knockdown of deubiquitinases was undertaken by small interfering RNA (siRNA). Results: The results of flow cytometric analysis indicated that the apoptotic rate of HSC-T6 was induced by I3C in a dose-dependent manner. Observation by acridine orange staining exhibited nuclear condensation and apoptotic bodies in I3C treated cells. Consistently, the expression ratio of $\mathrm{Bax} / \mathrm{bcl}-2$ was markedly increased by I3C. These results indicated that I3C could significantly induce apoptosis of HSC-T6 cells. Furthermore, Co-IP assay revealed K63 de-ubiquitination of RIP1 by I3C, associated with the induction of caspase 8. Although I3C had no effect on the expression of ubiquitin ligases cellular inhibitor of apoptosis 1/2 (cIAP1/2), the protein level of deubiquitinase cylindromatosis (CYLD) was significantly induced by I3C. Moreover, CYLD silencing reversed the pro-apoptosis induction effect of $\mathrm{I} 3 \mathrm{C}$ and reduced the expression ratio of Bax/bcl-2 in HSC-T6 cells. Conclusion: These results demonstrated that I3C could induce apoptosis of HSC through RIP1 K63 de-ubiquitination by upregulating deubiquitinase CYLD.

B. Li and M. Cong contributed equally to this work.

(C) 2017 The Author(s)

Published by S. Karger AG, Basel

Hui Wang and Ying Ao

Department of Pharmacology, School of Basic Medical Science of Wuhan University, Wuhan 430071, Hubei Province (China)

E-Mail wanghui19@whu.edu.cn / yingao@whu.edu.cn
\end{abstract}




\section{Cellular Physiology Cell Physiol Biochem 2017;41:1481-1490

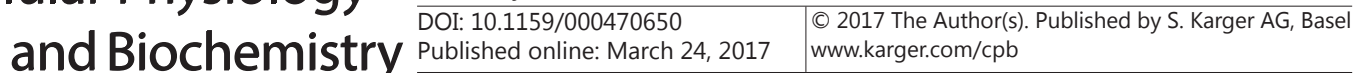 \\ Li et al.: Indole-3-Carbinol Induces Apoptosis of Hepatic Stellate Cells}

\section{Introduction}

Liver fibrosis is a common excessive healing response to various chronic liver injury, such as viral hepatitis, alcoholic hepatitis, nonalcoholic steatohepatitis, autoimmune hepatitis, and metabolic disease [1]. It is characterized by deposition of extracellular matrix (ECM) with distortion of normal hepatic parenchyma, eventually leading to dense fibrosis, cirrhosis, and development of portal hypertension [2]. Hepatic stellate cells (HSCs) are the primary cellular source of matrix components, and play a vital role in the development and maintenance of liver fibrosis [3-6]. In their quiescent state, HSCs store vitamin A and retinoid, but upon tissue damage, they become activated and undergo proliferation and secretion of ECM proteins such as collagen, glycoprotein, and proteoglycans leading to hepatic fibrosis [6]. It is well known that liver fibrosis is a reversible disease, with the apoptosis of activated HSCs being the central event in this reversal [7]. Therefore, induction the apoptosis of HSC is a potential direction for the treatment of live fibrosis.

Tumor necrosis factor (TNF)- $\alpha$ contributes to many physiological and pathological processes and plays an important role in mediating survival signaling and apoptosis. On TNF- $\alpha$ binding, TNF receptor 1 (TNFR1) undergoes a conformational change to form TNFR complex I containing TNFR1-associated death domain (TRADD), receptor-interacting protein (RIP)1 and TNF receptor-associated factor 2 (TRAF2). RIP1 K63 ubiquitination by cellular inhibitor of apoptosis $1 / 2$ (cIAP1/2) or TRAF2 can recruit transforming growth factor- $\beta$-activated kinase 1 (TAK1) to initiate the canonical NF- $\kappa B$ survival pathway $[8,9]$. On the other hand, upon inhibition of NF- $\kappa B$ signaling, for example, by K63 de-ubiquitination of RIP1 through deubiquitinase or loss of cIAPs, complex II containing TRADD, Fas-associated death domain (FADD), caspase 8 and RIP1 is formed and executes apoptosis which is triggered by caspase 8 [8]. Thus, the state of RIP1 K63 ubiquitination is a switch of TNF- $\alpha$ signaling pathway to determine the cell fate: survival or death [10].

Indole-3-carbinol (I3C), a naturally occurring compound generated from the hydrolysis of glucobrassicin, is found at exceptionally high concentrations in Brassica vegetables [11]. It has shown that I3C exerts a variety of beneficial effects which include anti-cancer [12], anti-microbial [13], immunomodulatory [14], and anti-inflammatory properties [15]. In our previous study, we have demonstrated that I3C protected liver slices from acetaldehydeinduced HSC activation [16]. In addition, it reversed liver fibrosis in rats induced by compound ( $\mathrm{CCl}_{4}$, ethanol and lipid) [17] or immunologic (porcine serum) factors [18] in vivo. Furthermore, we proved that I3C remarkably inhibited the proliferation of HSC in vitro [19]. Given the tight relationship with HSC apoptosis and reversion of liver fibrosis, we then aimed to elucidate the effect of I3C on the apoptosis of HSC and the underlying mechanism. In this study, we showed that I3C significantly induced the apoptosis of HSC in vitro, and the inhibition of RIP1 K63 ubiquitination was involved in the mechanism.

\section{Materials and Methods}

\section{Chemicals and Reagents}

I3C, dimethyl sulfoxide (DMSO), and L-glutamine were purchased from Sigma (St. Louis, MO, USA). Dulbecco's modified Eagle's medium (DMEM) and fetal bovine serum (FBS) were provided by Invitrogen (Carlsbad, CA, USA). TRIzolR reagent was purchased from Life technologies (Carlsbad, CA, USA). PrimeScriptR RT reagent Kit with gDNA Eraser and SYBRR Premix Ex Taq ${ }^{\mathrm{TM}}$ Kit were purchased from Takara Biotechnology Co., Ltd. (Dalian, China). All oligonucleotide primers were synthesized by Sangon Biotech Co., Ltd. (Shanghai, China). The protein detection kit was from Bio-Rad Laboratories, Inc. (Hercules, CA, USA). The enhanced chemiluminescence kit (ECL) and goat anti-rabbit IgG were provided by Thermo Pierce Biotechnology Inc. (Rockford, IL, USA). Antibody of rat caspase 8 (sc-81656), cIAP1 (sc-1869), cIAP2 (sc7944), TRAF2 (sc-7187) and protein $A+G$ agarose beads were purchased from Santa Cruz Biotechnology, Inc. (Dallas, Texas, USA). Monoclonal antibody of rat A20 (\#5630), cylindromatosis (CYLD) (\#8462) and RIP1 (\#3493) were obtained from Cell Signaling Technology, Inc. (Danvers, MA, USA). Monoclonal antibody 


\section{Cellular Physiology Cell Physiol Biochem 2017;41:1481-1490 \begin{tabular}{l|l} 
and Biochemistry Published online: March 24, 2017 & $\begin{array}{l}\text { (c) } 2017 \text { The Author(s). Published by S. Karger AG, Basel } \\
\text { www.karger.com/cpb }\end{array}$
\end{tabular}}

Li et al.: Indole-3-Carbinol Induces Apoptosis of Hepatic Stellate Cells

of rat K63 ubiquitin (ab179434) was provided by Abcam Technology, Inc. (Cambridge, MA, USA). siRNA oligonucleotides against rat CYLD were purchased from Shanghai GenePharma Co. Ltd. (Shanghai, China). All chemicals and reagents were of analytical grade.

\section{Cell culture}

HSC-T6, an immortalized rat liver stellate cell line, was purchased from Procell Life Science Co., Ltd (Wuhan, China). Cells were maintained at $37{ }^{\circ} \mathrm{C}, 5 \% \mathrm{CO}_{2} /$ air in high glucose DMEM supplemented with either $10 \%$ (v/v) FBS, penicillin (100 units/ml), streptomycin $(100 \mu \mathrm{g} / \mathrm{ml}), 2 \mathrm{mM}$ L-glutamine. After 70\% of confluency, the cells were cultured with high glucose DMEM without FBS for 12 hours before the further treatment. For apoptosis analysis, HSC-T6 cells were treated for 24 hours with 25, 50 and $100 \mu \mathrm{M}$ of I3C, which was dissolved in $0.1 \%$ DMSO in media.

\section{Flow cytometry analysis of apoptosis}

AnnexinV-fluorescein isothiocyanate (FITC) / propidium iodide (PI) apoptosis detection kit (BD, San Jose, CA, United States) was used for the measurement of apoptosis. Briefly, after the treatment of I3C for 24 hours, the cells were trypsinized and washed with cooled PBS and resuspended with $100 \mu \mathrm{L}$ binding buffer per $10^{5}$ cells. Following incubation with $5 \mu \mathrm{L}$ Annexin V-FITC and $5 \mu \mathrm{L}$ PI solution in a dark room at room temperature for 15 minutes, $400 \mu \mathrm{L}$ binding buffer was added and shaken slightly. The samples were collected and $10^{4}$ cells were analyzed by a FACSort flow cytometer (BD Biosciences).

\section{Acridine orange cytochemistry staining}

The cells were seeded in 24-well dishes containing $1 \mathrm{~mL}$ culture medium. After 24 hours incubation of different doses of I3C, The cells were washed twice by PBS, then fixed by $95 \%$ alcohol for 10 minutes, and stained by $0.01 \%$ acridine orange for 5 minutes [20]. Morphological change was observed by an inverted fluorescence microscope (Olympus, Tokoyo, Japan).

\section{Real-time quantitative PCR}

After the treatment of I3C in 6-well plates, Total RNA was extracted using TRIzol ${ }^{\circledR}$ reagent according to the manufacturer's instructions. One $\mu \mathrm{g}$ RNA was treated with gDNA Eraser and reverse transcribed to cDNA according to the protocol of the PrimScript ${ }^{\circledR}$ RT reagent kit. Q-PCR was performed on an ABI Step One RT-PCR thermal cycler (ABI Stepone, NY, USA) using SYBR ${ }^{\circledR}$ Premix Ex Taq ${ }^{\mathrm{TM}}$ kit. The housekeeping gene, glyceraldehyde 3-phosphate dehydrogenase (GAPDH), was used as a quantitative control. The Ct value of fluorescent product was detected at the extension period and the expression of target genes was analyzed by applying the $2^{-\Delta \Delta C t}$ relative quantifcation method. The mRNA expression levels of the target genes were normalized to GAPDH values, and then presented as fold values relative to control group. Primers and annealing temperatures of q-PCR were listed in Table 1.

\section{Western blot}

After the treatment of I3C with different doses for 24 hours in 6-well plates, cells were trypsinized and collected. Protein was extracted using RIPA buffer [50 mM Tris-HCl (PH 7.4), $150 \mathrm{mM} \mathrm{NaCl,} \mathrm{1 \%} \mathrm{NP-40,}$ $0.1 \%$ SDS]. Homogenates were centrifuged at $4^{\circ} \mathrm{C}$ for 10 minutes at $14,000 \mathrm{~g}$, the supernatants aliquots were collected. Protein concentrations were determined using a protein assay kit. Aliquots of lysate were mixed

Table 1. Oligonucleotide primers and PCR conditions of rat. in quantitative real-time PCR. Bax, Bcl2-associated X protein; Bcl2, B-cell CLL/lymphoma 2; CYLD, cylindromatosis; GAPDH, glyceraldehyde phosphate dehydrogenase

\begin{tabular}{lllcc}
\hline Genes & Forwardprimer $\left(5^{\prime}-3^{\prime}\right)$ & Reverseprimer $\left(5^{\prime}-3^{\prime}\right)$ & Product(bp) & $\begin{array}{c}\text { Annealing } \\
\left({ }^{\circ} \mathrm{C}\right)\end{array}$ \\
\hline Bax & CTAGCAAACTGGTGCTCAA & TCCCGAAGTAGGAAAGGAG & 150 & 60 \\
Bc-2 & GAGGATTGTGGCCTTCTTT & ATCCCAGCCTCCGTTAT & 154 & 60 \\
CYLD & TGCCAGGTTGCTAAAATCTTG & CCTTTGGGTACTCTCAGGAGC & 158 & 60 \\
GAPDH & GCAAGTTCAATGGCACAG & GCCAGTAGACTCCACGACA & 140 & 63 \\
\hline
\end{tabular}




\section{Cellular Physiology Cell Physiol Biochem 2017;41:1481-1490 \begin{tabular}{ll|l} 
DOI: 10.1159/000470650 & $\begin{array}{l}\text { O 2017 The Author(s). Published by S. Karger AG, Basel } \\
\text { www.karger.com/cpb }\end{array}$ \\
\hline
\end{tabular}}

with $5 \times$ loading buffer containing 2-mercaptoethanol and boiled at $100^{\circ} \mathrm{C}$ for 5 minutes. Samples with equal amounts of protein $(30 \mu \mathrm{g})$ were resolved on $12 \%$ sodiumdodecyl sulfate polyacrylamide gel (SDS-PAGE) and then transferred to nitrocellulose membranes. Nonspecific binding was blocked by TBST (25 mM Tris$\mathrm{HCl}, 50 \mathrm{mM} \mathrm{NaCl}, 0.05 \%$ Tween-20) containing 5\% dry milk for 60 minutes at room temperature, followed by overnight incubation at $4^{\circ} \mathrm{C}$ with $1: 1000$ diluted primary antibodies of anti-rat cIAP1, anti-rat cIAP2, antirat TRAF2, anti-rat A20, anti-rat CYLD or anti-rat GAPDH. After 1 hour of incubation with HRP-conjugated secondary antibody (1:5000 dilution), the signal was detected using ECL reagents (Pierce, Cramlington, UK). To compare the relative densities of target proteins among the groups, samples were normalized to GAPDH values, and then presented as fold values relative to control group.

\section{Immunoprecipitation}

After the treatment of $100 \mu \mathrm{M}$ of I3C for 24 hours in $75 \mathrm{~cm}$ flasks, the cells were harvested and washed with PBS and then lysed in RIPA buffer on ice for 1 hour. The supernatants were collected and adjusted to the same concentration after centrifugation at $17000 \mathrm{~g}$ for 15 minutes. A $\%$ input sample was set aside, and either primary antibody of RIP1 $(8 \mu \mathrm{L})$ or normal immunoglobulin antibody was added to $150 \mu \mathrm{L}$ cell lysates and rotated overnight at $4^{\circ} \mathrm{C}$. Subsequently, $25 \mu \mathrm{L}$ protein $\mathrm{A}+\mathrm{G}$ agarose beads were added to the mixture and rotated at $4^{\circ} \mathrm{C}$ for $3 \mathrm{~h}$. The target protein and its complex were collected at $4000 \mathrm{rpm}$ for 5 minutes at $4^{\circ} \mathrm{C}$ and washed three times with RIPA buffer. Immunoprecipitated proteins were separated in a $10 \%$ SDSPAGE and transferred to nitrocellulose membrane. Membranes were blocked with 5\% dry milk in TBST and incubated with 1:1000 diluted primary antibody of anti-rat K63 ubiquitin. Specific signals were revealed by the ECL detection reagent.

SiRNA knockdown of CYLD gene

To knockdown CYLD expression, RNA interference technology was used. The sequences of the CYLD siRNA were GGACCUUUAUUAGCGGAGATT and UCUCCGCUAAUAAAGGUCCTT. A pair of non-specific oligonucleotides (nonsilencing control) was used as a negative control; the sequences of the control siRNA were UUCUCCGAACGUGUCACGUTT and ACGUGACACGUUCGGAGAATT. Prior to transfection, HSC-T6 was seeded in six-well plates at a density of $4 \times 10^{5}$ cells per well. Twenty-four hours later, cells were then transfected with $30 \mathrm{nM}$ CYLD siRNA or control oligonucleotides using Lipofectamine 3000 in Opti-MEM according to the manufacturer's protocol. The medium was exchanged for a fresh medium after 6 hours, and then the cells were treated with $100 \mu \mathrm{M}$ I3C. The mRNA of the cells was harvested after 24 hours.

\section{Statistics}

SPSS 17.0 (SPSS Science Inc., Chicago, Illinois) was used for data analysis. For the quantification of data, each assay was repeated at least 3 times independently. All presented measurement data was expressed as the mean \pm S.E.M. and was evaluated with one-way ANOVA followed by a post hoc Dunnett- $t$-test for comparison among multiple groups. Statistical significance was set at $P<0.05$.

\section{Results}

I3C induces apoptosis of HSC-T6 cells

In order to determine the effect of I3C on apoptosis of HSC-T6, first, we evaluated the apoptotic rate by flow cytometric analysis with Annexin V-FITC/ PI staining. As shown in Fig. $1 \mathrm{~A}$, cells were gated into lower right (LR) and upper right (UR) quadrants, which represented early (Annexin V(+)/PI(-)) and late apoptotic (Annexin V(+)/PI(+)) cells, respectively. Cells in lower left (LL) quadrants were considered to be alive and those in the upper left (UL) quadrants were considered to be necrotic. The extent of apoptosis was expressed as the sum total of the percentages in LR and UR quadrants. As in the panel e of Fig. 1A, the apoptosis rate was increased dose-dependently by $48.8 \%, 100.5 \%(P<0.05)$ and $124.0 \%(P<0.05)$ in 25, 50 and $100 \mu \mathrm{M}$ I3C treated groups, respectively, when compared with the control. Next, we detected the morphological change of the apoptotic cells using acridine orange staining, which is a nucleic acid selective metachromatic dye that differentially stains single-stranded nucleic acids orange and double- stranded nucleic acids green [21]. As shown in Fig. 1B, 


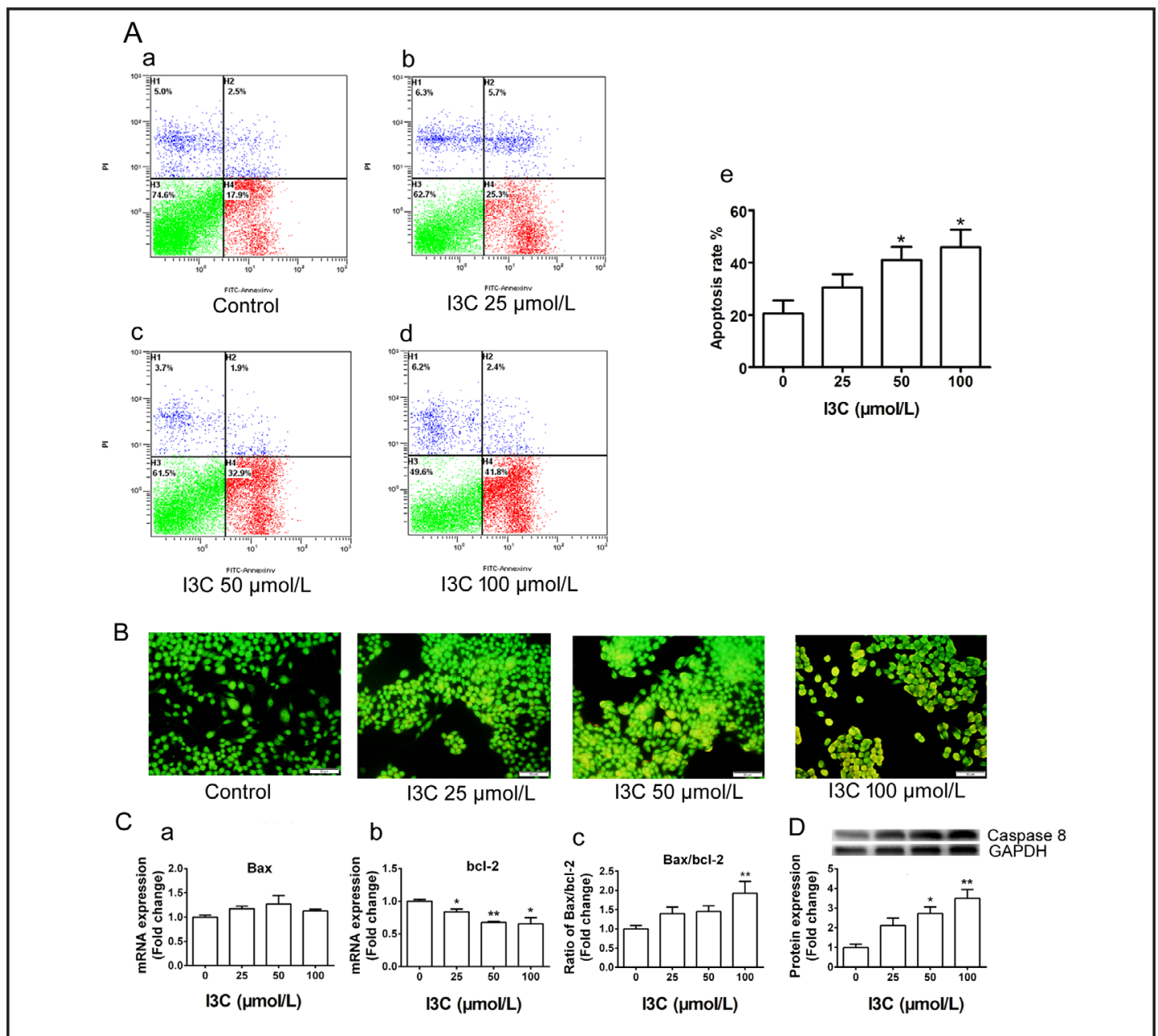

Fig. 1. Effects of I3C on apoptosis of HSC-T6 cells. A: HSCs apoptosis was determined by Annexin V/PI staining and analyzed by flow cytometry. Mean \pm S.E.M. $n=6$. B: HSC-T6 cells were stained by acridine orange. (magnification $\times 200$, bar $=50 \mu \mathrm{m}$ ) C: mRNA expression of Bax (a), bcl-2 (b) and the ratio of Bax/bcl-2 (c) in HSC-T6 cells were determined by q-PCR. The samples were normalized to GAPDH values, and then presented as fold values relative to control group. Mean \pm S.E.M., $n=6$. D: Protein expression of caspase 8 in HSC-T6 cells were determined by western blot. The samples were normalized to GAPDH values, and then presented as fold values relative to control group. Mean \pm S.E.M., $n=6$. $P$ value was calculated by one-way ANOVA followed by a post hoc Dunnett-t-test. ${ }^{*} P<0.05,{ }^{* *} P<0.01$ vs control.

Fig. 2. Effects of I3C on K63 ubiquitination of RIP1. The cells were treated with of $100 \mu \mathrm{M}$ of I3C for 24 hours in $75 \mathrm{~cm}$ flasks. Cells were lysed and endogenous RIP1 was pulled-down and the presence of ubiquitin was detected by western blot using antibodies specifically designed against K63 ubiquitin chains.

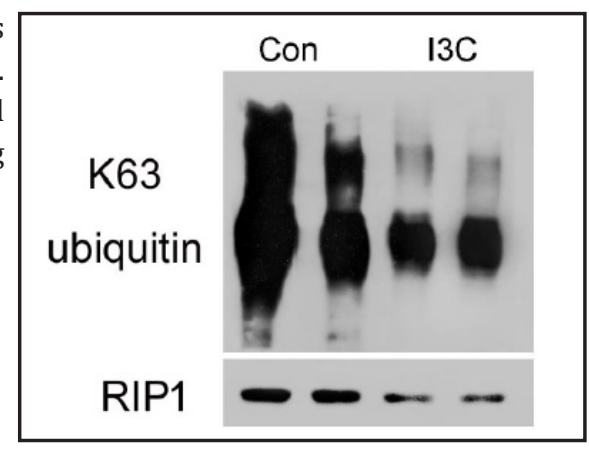

in I3C treated groups, the cells exhibited marked nuclear condensation and occurrence of apoptotic bodies, which indicated induction of apoptosis. Consistently, the result of semiquantitative analysis (Fig. 1C) showed that the expression of Bax, a pro-apoptotic gene, 


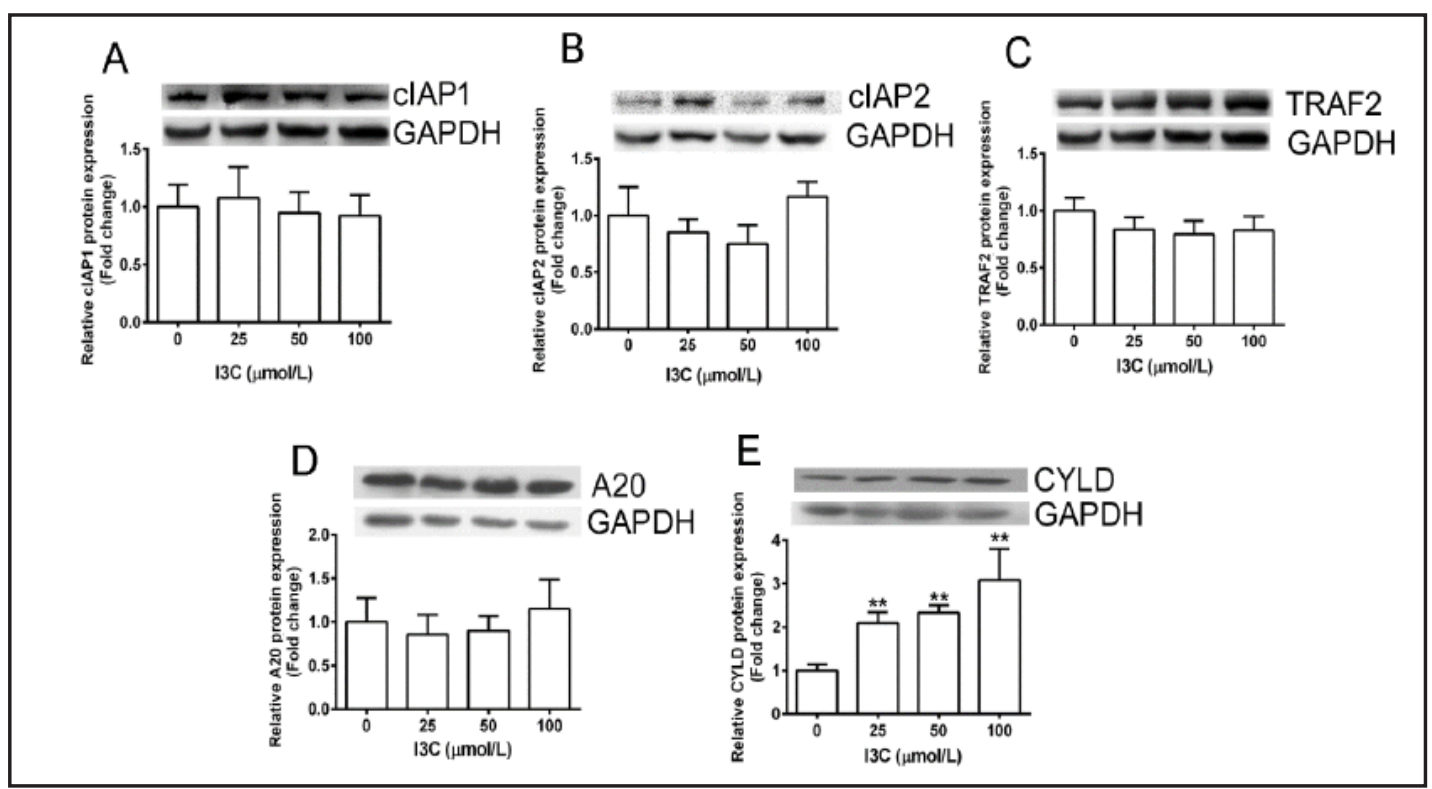

Fig. 3. Effect of I3C on the protein expression of RIP1 ubiquitination regulating enzymes. The cells were treated with of $100 \mu \mathrm{M}$ of I3C for 24 hours in 6-well plates. A-E: Protein expression of cIAP1 (A), cIAP2 (B), TRAF2 (C), A20 (D) and CYLD (E) in HSC-T6 cells was determined by western blot. The samples were normalized to GAPDH values, and then presented as fold values relative to control group. Mean \pm S.E.M., $n=$ 6. $P$ value was calculated by one-way ANOVA followed by a post hoc Dunnett- $t$-test. $* P<0.05, * * P<0.01$ vs control.

was increased, whereas the expression of bcl-2, an anti-apoptotic gene, was significantly reduced by I3C. Accordingly, the ratio of Bax/bcl-2 was increased by $40.0 \%, 45.2 \%$ and 92.9\% $(P<0.01)$ in 25,50 and $100 \mu \mathrm{M}$ I3C treated groups when compared with the control. Moreover, the protein expression of caspase 8 was enhanced by I3C with $112.3 \%, 174.6 \%$ $(P<0.05)$ and $251.4 \%(P<0.01$, Fig. 1D). Taken together, these results indicated that I3C could induce apoptosis of HSC-T6 cells in a dose-dependent manner.

\section{I3C inhibits K63 ubiquitination of RIP1}

Our previous study found that I3C inhibited the protein expression of NF- $\kappa \mathrm{B}$ pathway in HSC-T6 cells [22]. Since NF- $\kappa B$ pathway was activated by K63-ubiquitinated RIP1 [23, 24], In order to identify whether the inhibitory effect of I3C on NF- $\kappa B$ was due to modification of RIP1 ubiquitination status, we investigated K63-ubiquitination level of RIP1 in HSC-T6 cells. The results revealed that endogenous RIP1 was modified with K63 ubiquitin chains in control group, however, I3C treatment removed most of these ubiquitin chains (Fig. 2), which indicated that I3C could reduce RIP1 K63-ubiquitinated station in HSC-T6 cells.

\section{I3C induces the expression of deubiquitinases CYLD}

RIP1 K63 ubiquitination is regulated by ubiquitin ligases as well as deubiquitinases [23, 24]. In order to explore the mechanism of I3C on the modification of RIP1 K63 ubiquitinated state, we assayed the protein expression of ubiquitin ligases cIAP1, CIAP2, TRAF2 and deubiquitinases CYLD, A20. As shown in Fig. 3, no significant change was observed on the expression of cIAP1, CIAP2, TRAF2 or A20 among the groups (Fig. 3A-D), whereas CYLD protein level was increased by $109.5 \%(P<0.01), 133.0 \%(P<0.01)$ and $208.4 \%(P<0.01)$ in I3C treated groups when compared with control (Fig.3E). These results indicated that I3C could induce the expression of CYLD, which might be involved in the mechanism of I3C for modification of RIP1 K63-ubiquitination. 


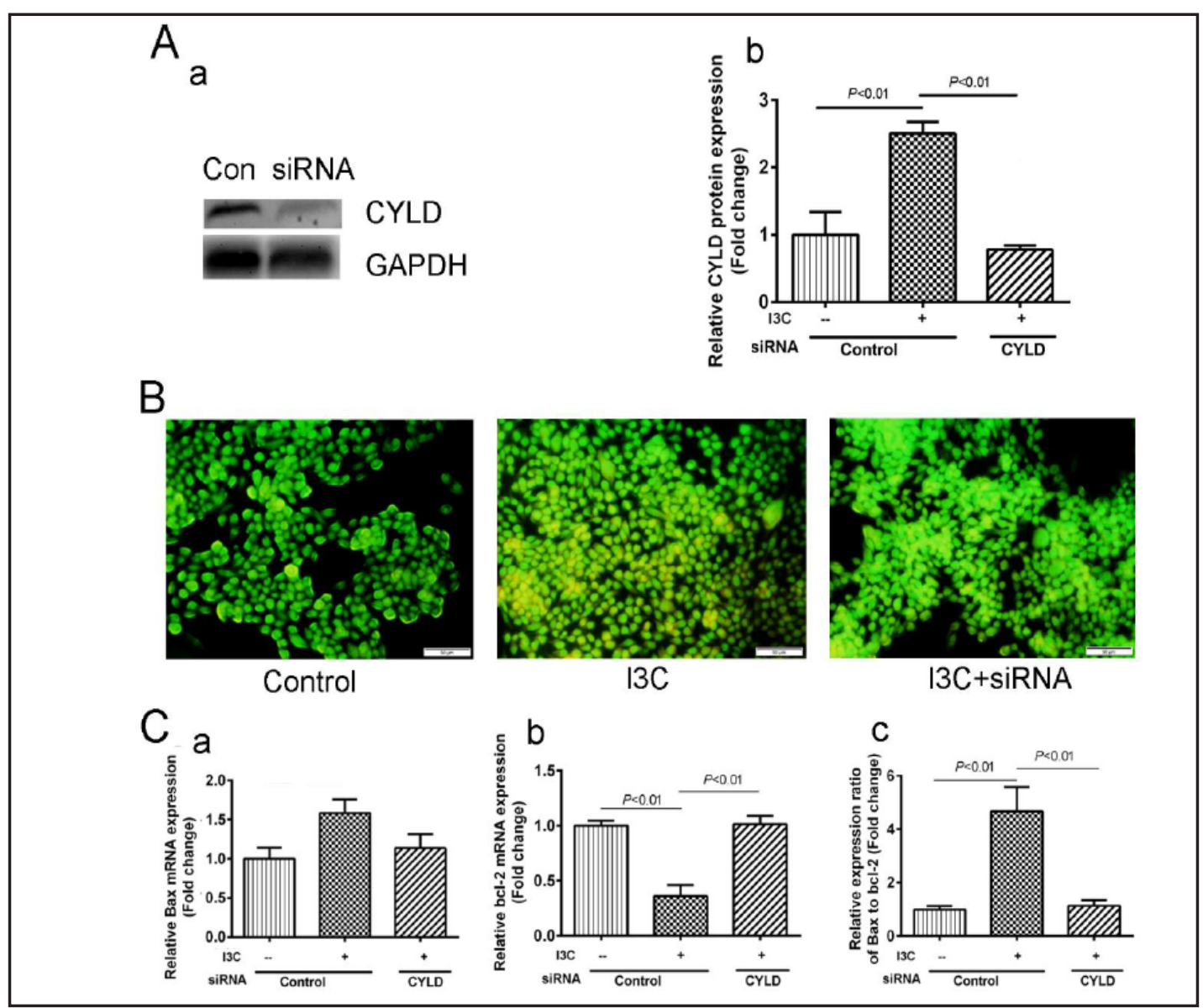

Fig. 4. Effect of CYLD knockdown on the pro-apoptotic effect of I3C. A: the protein expression of CYLD was detected by western blot (a) and the mRNA level was determined by q-PCR (b). The samples were normalized to GAPDH values, and then presented as fold values relative to control group. Mean \pm S.E.M., $n=6$. B: CYLD silenced cells were treated with $100 \mu \mathrm{M}$ of I3C for 24 hours. Cell apoptosis was determined by acridine orange staining. (magnification $\times 200$, , bar $=50 \mu \mathrm{m}$ ) C: mRNA expression of Bax (a), bcl-2 (b) and the ratio of Bax/bcl-2 (c) in HSC-T6 cells was determined by q-PCR. The samples were normalized to GAPDH values, and then presented as fold values relative to control group. Mean \pm S.E.M., $n=6$. $P$ value was calculated by one-way ANOVA followed by a post hoc Dunnett- $t$-test.

\section{CYLD silencing inhibits the apoptosis of HSC-T6}

In order to prove the involvement of CYLD on pro-apoptotic effect of I3C, we investigate the effect of CYLD silencing on the apoptosis of HSC-T6. As shown in Fig. 4A, the protein expression of CYLD was silenced after knockdown by siRNA (panel a), the induction effect of I3C on its gene expression was also reduced dramatically (panel b). Acridine orange staining showed that CYLD silencing reduced the apoptotic cells induced by I3C (Fig. 4B). In addition, knockdown of CYLD reversed the inhibition effect of I3C on bcl-2 gene expression as well as the induction on the ratio of Bax/ bcl-2 (Fig. 4C). All these results suggested that CYLD silencing could abolish the pro-apoptotic effect of I3C in HSC-T6.

\section{Discussion}

Proliferation and activation of HSCs is a key step in the development of liver fibrosis [2527]. It is reported that the extracellular signal-regulated kinases (ERK)/mitogen-activated protein kinase (MAPK) signaling pathway is involved in the proliferation of HSCs and the 
ECM synthesis in activated HSCs [26, 27]. Our previous study has indicated that I3C could inhibit the proliferation of HSC by blocking the NADPH oxidase/reactive oxygen species/ p38 MAPK signal pathway, which contributes to protective effect of I3C to liver fibrosis in rats [19]. Moreover, in our previous research in vivo, we also proved that I3C could reverse rat liver fibrosis in different models $[17,18]$, but the molecular mechanism remains unclear. Given that apoptosis of the activated HSC is the central event of the reversal of hepatic fibrosis [3-6], we speculated that I3C could also induce apoptosis of activated HSC. Thus, in this study, we investigated the effect of I3C on the apoptosis of HSC-T6 cells by the assays of flow cytometry analysis, acridine orange cytochemistry staining and expression of apoptotic regulating genes. As expected, all these results strongly suggested that I3C could significantly induce apoptosis of HSC.

TNF- $\alpha$ signaling pathway is an important death receptor-mediated extrinsic apoptosis pathway [28], which exerts both anti-apoptotic and pro-apoptotic effects [29]. After binding to TNF-R1, on one hand, TNF- $\alpha$ activates NF- $\kappa$ B pathway and inhibits apoptosis; on the other

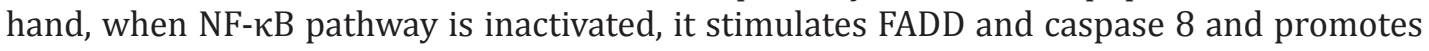
apoptosis [29]. In our previous study, the results of protein array analysis have indicated that I3C inhibited the protein expression of NF- $\mathrm{BB}$ pathway [22]. In this research, we found that I3C induced the protein expression of caspase 8 . Therefore, these results suggested that the inhibition to NF- $\kappa B$ pathway and the stimulation to caspase 8 might be involved in the proapoptotic effect of I3C.

In TNF- $\alpha$ signaling pathway, RIP1 is at the upstream of NF- $\kappa B$, its K63 ubiquitination state acts as a cell-death switch in response to TNF signaling [30]. K63 ubiquitinated RIP1 initiates NF- $\kappa$ B survival pathway, whereas de-ubiquitinated RIP1 is able to stimulate caspase 8 to trigger apoptosis. In the present study, we analyzed the K63 ubiquitination level in different groups, and the results indicated that I3C could inhibit RIP1 K63 ubiquitination state, which might be one of the mechanisms to repress NF- $\kappa B$ pathway and consequently trigger the expression of caspase 8 and apoptosis of HSC.

The K63 ubiquitination of RIP1 is mediated by ubiquitin ligases. TRAF2 serves as an adapter protein and recruits cIAP1 and 2 to the TNF-R1 signalosome, which then target RIP1 directly for polyubiquitination [31]. cIAP 1 and 2 are inhibitor of apoptosis protein family members, they show particularly strong structural homology and function as E3 ligases to mediate RIP1 K63 polyubiquitination [32]. However, in our study, I3C had no influence on the expressions of cIAP1/2 or TRAF2, which suggested that ubiquitin ligases might not be involved in repressive effect on RIP1 K63 ubiquitination by I3C. Like phosphorylation, ubiquitination is a reversible reaction being mediated by deubiquitinases. A20 and CYLD are two major de-ubiquitinating proteases that function in TNF- $\alpha$ signaling pathway [33, 34]. A20 is a bi-functional enzyme that acts on RIP1 both as an ubiquitin protease for K63linked ubiquitin chains and as an ubiquitin ligase that attaches K48 ubiquitinlinked chains, which promotes the degradation of RIP1 [35, 36]. CYLD was identified as a de-ubiquitinating protease that targets RIP1 and releases its K63-linked ubiquitin chains, thereby decreases the stability of NF- $\kappa B$ pathway as well as enhances cell death by promoting the formation of caspase 8 [37]. In our study, although I3C did not change the expression of A20, it stimulated the protein expression of CYLD in a dose-dependent manner, which suggested that the induction of CYLD contributes to the inhibition to RIP1 K63 ubiquitination by I3C. To further confirm this hypothesis, we knocked down CYLD in HSC-T6 cells to investigate the influence to the pro-apoptotic effect of I3C. Indeed, CYLD silencing inhibited apoptosis of HSC-T6 cells with the evidence of reduced apoptotic cells as well as increased expression ratio of Bax/ bcl-2. Thus, these results indicated that I3C inhibited RIP1 K63 ubiquitination and induced apoptosis of HSC-T6 cells through, at least partly, induction of CYLD.

Taken together, for the first time, the present study demonstrated that I3C could induce apoptosis of HSC through inhibition to RIP1 K63 ubiquitination. In brief, I3C upregulates CYLD expression, thereby degrades K63 ubiquitination of RIP1. Consequently, it inhibits NF$\kappa \mathrm{B}$ pathway and promotes caspase 8-triggered apoptosis in HSC. Thus, together with our previous study in vivo, we have proved that I3C could reverse liver fibrosis through inducing 


\section{Cellular Physiology Cell Physiol Biochem 2017;41:1481-1490

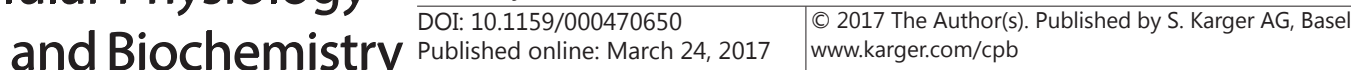

Li et al.: Indole-3-Carbinol Induces Apoptosis of Hepatic Stellate Cells

apoptosis of HSC, therefore, I3C might be a potential promising therapeutic drug for liver fibrosis treatment.

\section{Acknowledgement}

This work was supported by grants from the National Natural Science Foundation of China (No. 81001466).

\section{Disclosure Statement}

None.

\section{References}

1 Hernandez-Gea V, Friedman SL: Pathogenesis of liver fibrosis. Annu Rev Pathol 2011;6:425-456.

2 Bataller R, Brenner DA: Liver fibrosis. J Clin Invest 2005;115:209-218.

-3 Puche JE, Saiman Y, Friedman SL: Hepatic stellate cells and liver fibrosis. Compr Physiol 2013;3:14731492.

4 Friedman SL: Mechanisms of hepatic fibrogenesis. Gastroenterology 2008;134:1655-1669.

-5 Novo E, Cannito S, Paternostro C, Bocca C, Miglietta A, Parola M: Cellular and molecular mechanisms in liver fibrogenesis. Arch Biochem Biophys 2014;548:20-37.

6 Kisseleva T, Brenner DA: Hepatic stellate cells and the reversal of fibrosis. J Gastroenterol Hepatol 2006;21:S84-87.

7 Fallowfield JA: Therapeutic targets in liver fibrosis. Am J Physiol Gastrointest Liver Physiol 2011;300:G709715.

8 Wang JS, Wu D, Huang DY, Lin WW: TAK1 inhibition-induced RIP1-dependent apoptosis in murine macrophages relies on constitutive TNF-alpha signaling and ROS production. J Biomed Sci 2015;22:76.

-9 Bettermann K, Vucur M, Haybaeck J, Koppe C, Janssen J, Heymann F, Weber A, Weiskirchen R, Liedtke C, Gassler N, Muller M, de Vos R, Wolf MJ, Boege Y, Seleznik GM, Zeller N, Erny D, Fuchs T, Zoller S, Cairo S, Buendia MA, Prinz M, Akira S, Tacke F, Heikenwalder M, Trautwein C, Luedde T: TAK1 suppresses a NEMOdependent but NF-kappaB-independent pathway to liver cancer. Cancer Cell 2010;17:481-496.

10 Haas TL, Emmerich CH, Gerlach B, Schmukle AC, Cordier SM, Rieser E, Feltham R, Vince J, Warnken U, Wenger T, Koschny R, Komander D, Silke J, Walczak H: Recruitment of the linear ubiquitin chain assembly complex stabilizes the TNF-R1 signaling complex and is required for TNF-mediated gene induction. Mol Cell 2009;36:831-844.

11 Busbee PB, Nagarkatti M, Nagarkatti PS: Natural indoles, indole-3-carbinol (I3C) and 3,3'-diindolylmethane (DIM), attenuate staphylococcal enterotoxin B-mediated liver injury by downregulating miR-31 expression and promoting caspase-2-mediated apoptosis. PLoS One 2015;10:e0118506.

12 Ahmad A, Sakr WA, Rahman KM: Anticancer properties of indole compounds: mechanism of apoptosis induction and role in chemotherapy. Curr Drug Targets 2010;11:652-666.

13 Sung WS, Lee DG: In vitro antimicrobial activity and the mode of action of indole-3-carbinol against human pathogenic microorganisms. Biol Pharm Bull 2007;30:1865-1869.

14 Exon JH, South EH: Dietary indole-3-carbinol alters immune functions in rats. J Toxicol Environ Health A 2000;59:271-279.

15 Rouse M, Singh NP, Nagarkatti PS, Nagarkatti M: Indoles mitigate the development of experimental autoimmune encephalomyelitis by induction of reciprocal differentiation of regulatory T cells and Th17 cells. Br J Pharmacol 2013;169:1305-1321.

16 Wang H, Guo Y, Wu XQ Zhang C, Liao ZX: Effect of indole-3-carbinol on ethanol-induced liver injury and acetaldehyde-stimulated hepatic stellate cells activation using precision-cut rat liver slices. Clin Exp Pharmacol Physiol 2012;39:642. 


\section{Cellular Physiology Cell Physiol Biochem 2017;41:1481-1490 \begin{tabular}{ll|l} 
DOI: 10.1159/000470650 & Ond Biochemistry 2017 The Author(s). Published by S. Karger AG, Basel \\
wwww.karger.com/cpb
\end{tabular}

17 Gao AM, Ping J, Wang H. Therapeutic effects of indole-3-carbinol on multiple hepatotoxic factors-induced liver fibrosis in rats. Chin Pharmacol Bull 2011;27:764-769.

18 Ping J, Gao AM, Xu D, Li RW, Wang H. Therapeutic effects of indole-3-carbinol on pig serum-induced liver fibrosis in rats. Acta Pharm Sin 2011;46:915-921.

-19 Ping J, Li JT, Liao ZX, Shang L, Wang H: Indole-3-carbinol inhibits hepatic stellate cells proliferation by blocking NADPH oxidase/reactive oxygen species/p38 MAPK pathway. Eur J Pharmacol 2011;650:656-662.

20 Li L, Zhao D, Li J: Alteration of apoptotic protease activating factor 1 expression and possible role in ONOO(-)-induced apoptosis in human cerebral vascular smooth muscle cells. Int J Clin Exp Med 2015;8:19739-19745.

21 Yang SM, Tsai KD, Wong HY, Liu YH, Chen TW, Cherng J, Hsu KC, Ang YU, Cherng JM: Molecular Mechanism of Cinnamomum verum Component Cuminaldehyde Inhibits Cell Growth and Induces Cell Death in Human Lung Squamous Cell Carcinoma NCI-H520 Cells In Vitro and In Vivo. J Cancer 2016;7:251-261.

22 Wei XN, Li XH, Qin HQ Ao Y, Shen L, Ping J, Wang H. Molecular mechanisms of anti-fibrotic effect of indole3-carbinol by protein array asays. Med J Wuhan Univ 2010;31:709-712.

23 de Almagro MC, Goncharov T, Newton K, Vucic D: Cellular IAP proteins and LUBAC differentially regulate necrosome-associated RIP1 ubiquitination. Cell Death Dis 2015;6:e1800.

24 Wu P, Shi KJ, An JJ, Ci YL, Li F, Hui KY, Yang Y, Xu CM: The LEF1/CYLD axis and cIAPs regulate RIP1 deubiquitination and trigger apoptosis in selenite-treated colorectal cancer cells. Cell Death Dis 2014;5:e1085.

25 Li G, Li J, Li C, Qi H, Dong P, Zheng J, Yu F: MicroRNA-125a-5p Contributes to Hepatic Stellate Cell Activation through Targeting FIH1. Cell Physiol Biochem 2016;38:1544-1552.

26 Huang Q Liang C, Wei L, Nie J, Lu S, Lu C, Zhuo L, Lu Z, Lin X: Raf Kinase Inhibitory Protein DownExpression Exacerbates Hepatic Fibrosis In Vivo and In Vitro. Cell Physiol Biochem 2016;40:49-61.

-27 Lin X, Bai F, Nie J, Lu S, Lu C, Zhu X, Wei J, Lu Z, Huang Q: Didymin Alleviates Hepatic Fibrosis Through Inhibiting ERK and PI3K/Akt Pathways via Regulation of Raf Kinase Inhibitor Protein. Cell Physiol Biochem 2016;40:1422-1432.

28 Tsuchiya Y, Nakabayashi O, Nakano H: FLIP the Switch: Regulation of Apoptosis and Necroptosis by cFLIP. Int J Mol Sci 2015;16:30321-30341.

29 Elsharkawy AM, Oakley F, Mann DA: The role and regulation of hepatic stellate cell apoptosis in reversal of liver fibrosis. Apoptosis 2005;10:927-939.

30 O'Donnell MA, Legarda-Addison D, Skountzos P, Yeh WC, Ting AT: Ubiquitination of RIP1 regulates an NFkappaB-independent cell-death switch in TNF signaling. Curr Biol 2007;17:418-424.

- 31 Yin Q, Lamothe B, Darnay BG, Wu H: Structural basis for the lack of E2 interaction in the RING domain of TRAF2. Biochemistry 2009;48:10558-10567.

- 32 Liston P, Roy N, Tamai K, Lefebvre C, Baird S, Cherton-Horvat G, Farahani R, McLean M, Ikeda JE, MacKenzie A, Korneluk RG: Suppression of apoptosis in mammalian cells by NAIP and a related family of IAP genes. Nature 1996;379:349-353.

33 Lee EG, Boone DL, Chai S, Libby SL, Chien M, Lodolce JP, Ma A: Failure to regulate TNF-induced NF-kappaB and cell death responses in A20-deficient mice. Science 2000;289:2350-2354.

-34 Sun SC: CYLD: a tumor suppressor deubiquitinase regulating NF-kappaB activation and diverse biological processes. Cell Death Differ 2010;17:25-34.

-35 Ramkhelawon B, Hennessy EJ, Menager M, Ray TD, Sheedy FJ, Hutchison S, Wanschel A, Oldebeken S, Geoffrion M, Spiro W, Miller G, McPherson R, Rayner KJ, Moore KJ: Netrin-1 promotes adipose tissue macrophage retention and insulin resistance in obesity. Nat Med 2014;20:377-384.

-36 Ning S, Pagano JS: The A20 deubiquitinase activity negatively regulates LMP1 activation of IRF7. J Virol 2010;84:6130-6138.

37 Kupka S, Reichert M, Draber P, Walczak H: Formation and removal of poly-ubiquitin chains in the regulation of tumor necrosis factor-induced gene activation and cell death. FEBS J 2016;283:2626-39. 